\title{
Comparison of the new miniature Wright peak flow meter with the standard Wright peak flow meter
}

\author{
H G OLDHAM,* M M BEVAN, AND M McDERMOTT \\ From the MRC Pneumoconiosis Unit, Llandough Hospital, Penarth, South Glamorgan \\ CF6 IXW, UK
}

ABSTRACT Preproduction and current models of the miniature Wright peak flow meter have been compared with the standard Wright peak flow meter on normal and abnormal subjects. Early problems in production appear to have been overcome, and the current model agrees to within $3 \%$ with the standard peak flow meter, which is as close as the agreement between two standard instruments. The new mini-meter may be enclosed in a case, making direct comparisons with other instruments possible.

A new miniature Wright peak flow meter (minimeter) has been designed to meet the need for a simple, lightweight, inexpensive instrument for clinical use, particularly for self-monitoring by patients (Wright, 1978). The aim of this study was to compare its performance with that of the standard Wright peak flow meter (PFM) (Wright and McKerrow, 1959).

\section{Methods}

TESTING OF EARLY MODELS

The first mini-meters to be tested were preproduction models in 1976, which were compared with a standard PFM by asking subjects to blow into each instrument. Figure 1 shows the discrepancy between the mini-meter and the PFM, and changes to the scale were recommended to correct this. After the scale had been modified the instrument went into production, and two batches of the production models were tested in 1977 and early 1978. Unfortunately, despite the modified scale, the discrepancy between the minimeter and PFM still existed. This was also observed by Perks et al (1979), whose results agreed very closely with the data shown in fig 1 . The manufacturers then discovered a fault in the tension spring, which they subsequently corrected, and the model with modified scale and spring is now in production.

*Present address : Department of Pharmacy and Pharmacology, Bath University, Claverton Down, Bath BA2 7AY.

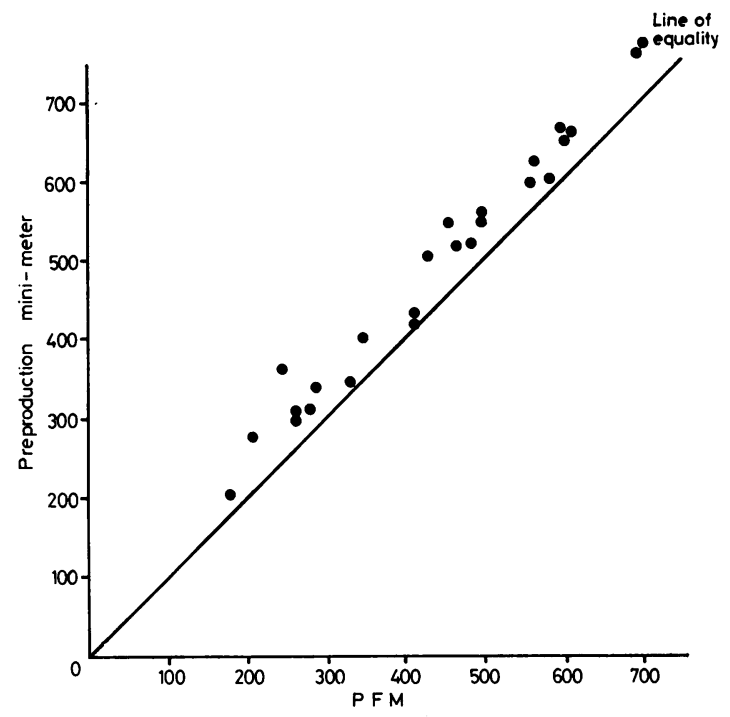

Fig 1 Comparison of peak flows measured using a preproduction mini-meter with those measured using a standard PFM. Subjects blew into each instrument separately.

TESTING OF CURRENT MODEL

During August 1978, six of the mini-meters currently in production were tested using steady flow. Of these, one had a mechanical fault in the valve at the mouthpiece end while the other five had similar, satisfactory performances. One of these five was compared with a standard PFM and with a preproduction mini-meter. It is obviously an 
advantage if two instruments can be directly compared by measuring the same blows; to achieve this, a perspex case was constructed in which a mini-meter could be enclosed allowing it to be connected in series with the PFM or with another mini-meter (fig 2). The steady-flow performance was tested with the mini-meter enclosed in the case. The subjects who took part in the study were volunteers, and included normal subjects, patients with chronic lung disease and children, both normal subjects and patients, so that the full range of the instrument was covered.

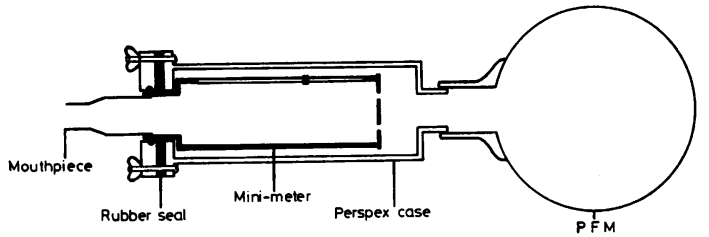

Fig 2 Mini-meter connected in series with PFM.

\section{PLAN OF STUDY}

The comparisons with the PFM and with the preproduction mini-meter were carried out on separate occasions, using the same procedure each time. The subject was asked to blow twice into the two instruments connected in series, and twice into each instrument separately, so that the effect of connecting them in series could be determined and the order of blows was statistically balanced to counteract the effects of learning and fatigue. Forty-seven subjects took part in the comparison with the PFM and 44 in the comparison with the preproduction mini-meter, many of them taking part in both comparisons. All three instruments were tested with steady-flow before and after the study.

\section{Results}

The steady-flow performance of all three instruments was unchanged at the end of the study. In the case of the PFM and the preproduction minimeter, it had not changed since the 1976 study, while for the current model it was unaffected by enclosing the instrument in the perspex case. Figures 3 and 4 show the comparisons on subjects of the mini-meter with the preproduction minimeter and with the standard PFM, the instruments being connected in series in both cases. The effect of the modifications to the scale and spring is clearly shown in fig 3 . It can be seen from fig 4 that the mini-meter now agrees much more closely with the PFM than did the preproduction model shown in fig 1 . Straight lines were fitted to the

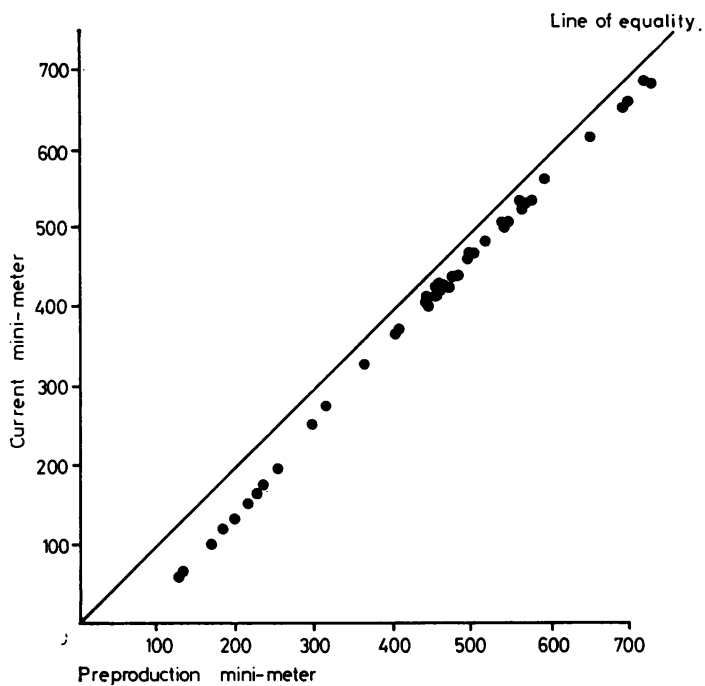

Fig 3 Comparison of peak flows measured using a current mini-meter with those measured using $a$ preproduction mini-meter. The two instruments were connected in series.

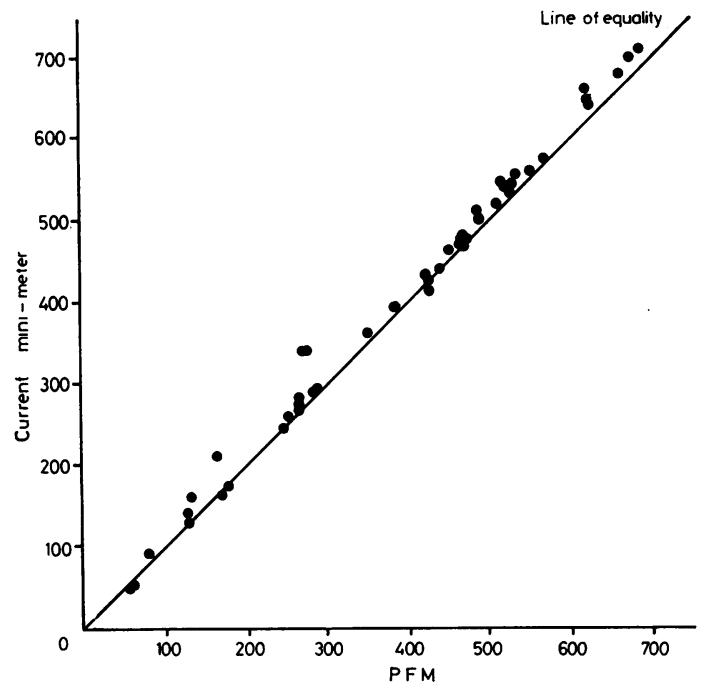

Fig 4 Comparison of peak flows measured using a current mini-meter with those measured using a standard PFM. The two instruments were connected in series.

data by calculating the regression of the difference of each pair of readings on their sum (the orthogonal regression). For the data in fig 1 the equation of this line is:

Preproduction mini-meter $=1.025 \times$ PFM +36.9 whereas for the data in fig 4 its equation is:

Current mini-meter $=1.020 \times \mathrm{PFM}+4.9$ 
In the latter case the intercept has been considerably reduced and does not differ significantly from zero, so that the equation of the orthogonal regression line through the origin may be calculated. Its equation is:

$$
\text { Mini-meter }=1.030 \times \mathbf{P F M}
$$

which shows that the mini-meter reads $3 \%$ higher than the PFM.

The readings obtained when the mini-meter and PFM are connected in series are about $2 \%$ lower than those obtained when they are blown into separately. The relationship, however, between the instruments is unchanged, since when they are blown into separately, the equation of the orthogonal regression line through the origin is:

$$
\text { Mini-meter }=1.028 \times \text { PFM }
$$

Connecting them in series produces a correlation coefficient of 0.996 , which is a material improvement on the figure of 0.992 obtained when they are used separately.

\section{Discussion}

The early problems in the production of the minimeter appear to have been successfully overcome and the current instrument agrees well with the standard peak flow meter. The mini-meter used in this study differs from the peak flow meter by only $3 \%$ with a correlation coefficient of 0.996 , an agreement which is as close as one would expect to find between two standard peak flow meters.

The fact that the mini-meter can be enclosed in a case and connected in series with another meter means that much more precise comparisons can be made with other instruments. The classic procedure of alternate peak flows into two instruments, though inevitable in the past, means that the correlation coefficient depends on the repeatability of the peak flows from each subject. The new procedure is therefore a substantial advance in this field as any new peak flow meter can be compared directly with a mini-meter using virtually any subject, including those who can manage only one peak flow at a time.

We thank Dr P D Oldham for statistical advice, Mr M F Clay who did the drawings, and Mrs P E McCarthy for advice and criticism.

\section{References}

Perks, W H, Tams, I P, Thompson, D A, and Prowse, K (1979). An evaluation of the mini-Wright peak flow meter. Thorax, 34, 79-81.

Wright, B M (1978). A miniature Wright peak-flow meter. British Medical Journal, 2, 1627-1628.

Wright, B M, and McKerrow, C B (1959). Maximum forced expiratory flow rate as a measure of ventilatory capacity. British Medical Journal, 2, 10411047. 